\title{
NOUVELle
}

\section{La crête neurale embryonnaire construit notre identité faciale}

\section{Le visage des vertébrés}

Gérard Couly ${ }^{1,2}$, lisabeth Dupin ${ }^{1}$

\author{
${ }^{1}$ Sorbonne Universités, UPMC Université Paris 06, Institut de la \\ vision, Inserm UMRS 968, 17, rue Moreau, 75012 Paris, France. \\ ${ }^{2}$ Université Paris Descartes-Sorbonne Paris Cité, Hôpital \\ Necker-Enfants malades, Assistance publique-Hôpitaux de \\ Paris, 149, rue de Sèvres, 75015 Paris, France. Professeur \\ honoraire de chirurgie maxillo-faciale de l'enfant. \\ gerard.couly@gmail.com
}

> Notre visage est reconnu par nos congénères grâce à certaines caractéristiques morphologiques qui lui confèrent son identité. II abrite les organes récepteurs de quatre de nos cinq sens (vision, olfaction, gustation, audition) dont les nerfs se projettent sur des aires dédiées du cerveau. II peut donc être considéré comme l'assistant sensoriel du cerveau [1]. Le visage est aussi l'acteur de notre communication expressive, de notre langage, et de nos émotions : il expose notre intimité psychique.

Pour comprendre la construction de notre visage, et ainsi son identité et sa relation directe avec le cerveau, il faut se reporter aux acquis de la recherche en embryologie crânio-faciale des cinquante dernières années. Le visage se construit, chez l'embryon humain comme chez celui de tous les vertébrés, grâce aux cellules de la crête neurale céphalique. À partir de 1968, les travaux de recherche à I'Institut d'embryologie cellulaire et moléculaire du CNRS et du Collège de France, dirigé par Nicole Le Douarin, ont permis de découvrir les propriétés biologiques et génétiques de la crête neurale chez l'embryon d'oiseau, vertébré dont le développement est proche de celui des mammifères, grâce à la construction de chimères entre les embryons du poulet et de la caille [2]. Ces travaux ont démontré les contributions de ces cellules au développement de la face et de nombreux organes [2]. La crête neurale est une structure embryonnaire transitoire, commune à tous les vertébrés. Elle s'individualise à partir du feuillet superficiel, l'ectoderme, au stade neurula, où apparaissent les premiers signes visibles du développement du système nerveux (Figure 1). Les cellules qui constituent la crête neurale migrent depuis la surface dorsale du tube neural (destiné à devenir le cerveau et la moelle épinière), telles «une vague cellulaire » qui déferlerait dorso-ventralement de chaque côté du plan de symétrie de l'embryon, d'abord vers le futur visage, puis vers le cou et le tronc. Ces cellules migrent dans l'embryon de façon invasive. Au cours de leur voyage, et lorsqu'elles sont arrivées à destination, elles échangent avec d'autres cellules des signaux moléculaires qui conditionnent leur différenciation finale en des types cellulaires variés [2].

Comme chez tous les vertébrés, la construction du visage humain est modulaire: lors du $1^{\mathrm{e}}$ mois de gestation, les organes sensoriels localisés dans le visage sont les premiers à se développer à partir d'épaississements du revêtement ectodermique appelés «placodes » [1]. Puis, au cours du $2^{\mathrm{e}}$ mois, la migration et la prolifération des cellules de la crête neurale céphalique vers et autour de ces placodes assurent le développement volumique des cinq modules préfigurant la face : un module naso-frontal, en position médiane supérieure, deux modules maxillaires et deux modules mandibulaires latéraux, droits et gauches. À la fin du $2^{\mathrm{e}}$ mois, ces modules, qui délimitent la cavité buccale, fusionnent par apoptose de l'ectoderme de leur surface : le visage acquiert alors «figure humaine». La défaillance de ce processus d'apoptose laisse en place des espaces entre les modules, dont les manifestations cliniques les plus fréquentes sont les fentes labio-maxillaires et palatines [3].

Les travaux de Le Douarin et al. [4] ont montré que le squelette et la majorité des autres tissus qui composent la face proviennent de la crête neurale de l'embryon qui dérive du feuillet ectodermique, alors que, dans le reste du corps, os, muscles, et tissus conjonctifs ont une origine embryologique différente et proviennent du feuillet mésodermique. La crête neurale a joué un rôle majeur dans la complexification de la tête au cours de l'évolution des vertébrés, en assurant la protection de leurs organes sensoriels et de leur cerveau par un squelette facial osseux. Cette ébauche embryonnaire est aussi à l'origine des éléments qui ont permis l'apparition de la respiration nasale et d'un appareil masticateur denté nécessaire à la prédation orale. À la lumière de ces résultats, Gans et Northcutt [5] ont proposé l'idée que la crête neurale a joué un rôle déterminant au cours de l'évolution dans la construction de la «nouvelle tête » des vertébrés.

Depuis trente ans, la mise en évidence de nouveaux types cellulaires faciaux produits par la crête neurale céphalique, en particulier lors de la construction du segment antérieur des yeux [6] et de celle des dents [7], est venue enrichir nos connaissances. La crête neurale céphalique est également fondamentale pour le développement du cerveau antérieur (ou télencéphale) et ses méninges. Ainsi, les expériences d'excision précoce des cellules de la crête neurale céphalique avant leur migration, réalisées par Le Douarin et al. [8] entre 2000 et 2004, ont abouti à la disparition des dérivés squelettiques et 


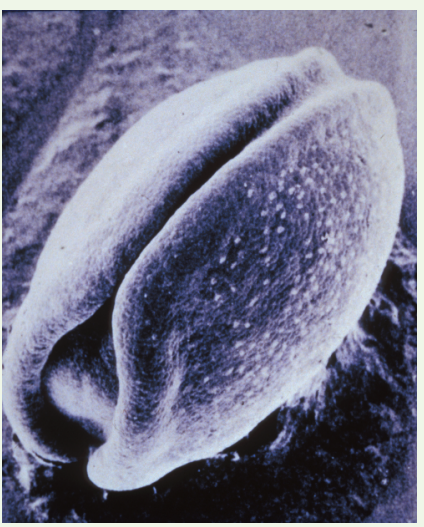

Figure 1. Début du développement de l'ébauche neurale. Neurula d'amphibien crête neurale (ㄷ Inserm - Nicole Le Douarin).

conjonctifs de la face, ainsi qu'à l'absence du télencéphale, du thalamus et du tectum optique, associée au défaut d'expression des gènes caractérisant la région cérébrale antérieure et dorsale. Les cellules de la crête neurale céphalique ont ainsi une double fonction: construire le visage et contribuer au développement du cerveau antérieur. Cette double fonction souligne l'importance morphogénétique de cette structure transitoire de l'embryon.

La liste des tissus et organes de la face dérivés de la crête neurale céphalique est la suivante [2] :

- Le squelette ostéo-cartilagineux facial : les os membraneux et les cartilages de l'appareil masticateur, de l'orbite, du crâne (frontal, pariétal, temporal), la chaîne des osselets de l'oreille moyenne et les cartilages de l'ethmoïde, du nez, et des pavillons de l'oreille.

- La dentine et les tissus de soutien des dents.

- Le tissu conjonctif de la face : derme, tissu cellulaire sous-cutané, graisse.

- Les cellules pigmentaires (mélanocytes), qui donnent leur couleur spécifique à la peau, aux poils, cheveux, et sourcils.

- Les muscles peauciers responsables des expressions du visage.

- Les cellules de Schwann des nerfs faciaux et les neurones des ganglions crâniens sensoriels et autonomes.
- Le tissu conjonctif et les lames tendineuses et aponévrotiques des muscles masticateurs, linguaux, et oculomoteurs. - Le segment antérieur des yeux : stroma et épithélium interne de la cornée, sclérotique, choroïde, muscles et cellules pigmentaires de l'iris, et muscle ciliaire. - Les parois musculaires des vaisseaux sanguins de la face et du cerveau.

La face est donc construite chez l'embryon exclusivement par les cellules de la crête neurale céphalique, à l'exception des cellules épidermiques dont l'origine est l'ectoderme non neural, de l'endothélium vasculaire, et des myocytes des muscles masticateurs, linguaux, et oculomoteurs, qui dérivent du mésoderme [2, 4].

Alors que les cellules de la crête neurale céphalique se différencient dans les modules de la face en produisant une très grande variété de types cellulaires (cellules neurales, mélanocytaires, et mésenchymateuses, constitutives notamment de nombreux dérivés conjonctivo-squelettiques), les éléments dérivés de la crête neurale troncale se limitent aux neurones sensoriels et autonomes, aux cellules gliales de Schwann, et aux cellules pigmentaires [2, 9]. Cette situation peut être corrélée aux expressions géniques différentes de la crête neurale de ces deux territoires : les cellules issues de la crête neurale céphalique, à destinée faciale, n'expriment pas de gènes architectes de la famille $H O X^{l}$, alors que les cellules de la crête neurale troncale expriment un ou plusieurs de ces gènes selon un code («code HOX») caractéristique de leur position le long des axes rostro-caudal du corps et proximo-

$(\rightarrow)$ Voir la Synthèse de F. Jacob, $m / s n^{\circ} 2$, distal des membres février 1994, page 145 [10] $(\rightarrow)$.

Si l'expression de gènes HOX est induite expérimentalement dans la crête neurale céphalique, le développement ultérieur de la face et du cerveau antérieur est sévèrement compromis [8].

\footnotetext{
${ }^{1}$ Les gènes Hox font partie des gènes homéotiques et sont
} présents chez tous les animaux à symétrie bilatérale.
La crête neurale céphalique embryonnaire, en produisant la quasi-totalité des types cellulaires entrant dans l'édification de notre face, construit l'identité du visage de chaque être humain. Ainsi, la crête neurale céphalique a fait plus que construire la nouvelle tête des vertébrés au cours de l'évolution; elle a aussi largement contribué à spécifier l'identité de leurs visages. $\diamond$

The embryonic neural crest builds our facial identity. The vertebrate face

\section{LIENS D'INTÉRÊT}

Les auteurs déclarent n'avoir aucun lien d'intérêt concernant les données publiées dans cet article.

\section{RÉFÉRENCES}

1. Belle M, Godefroy D, Couly G, et al. Tridimensional visualization and analysis of early human development. Cell 2017 ; 169 : 161-73.

2. Le Douarin NM, Kalcheim C. The neural crest, $2^{\text {nd }}$ ed. New York, Cambridge: University Press, 1999 : 472 p.

3. Ferreti $\varepsilon$, Bingsi L, Zewdu R, et al. A conserved Pbx-Wnt-P63-Irf6 regulatory module controls face morphogenesis by epithelial apoptosis. Dev Cell 2011 $21: 627-41$.

4. Couly G, Coltey P, Le Douarin NM. The triple origin of skull in higher vertebrates: a study in quail-chick chimeras. Development 1993 ; 117 : 409-29.

5. Gans C, Northcutt RG. Neural crest and the origin of vertebrates: a new head. Science $1983 ; 220: 268-74$.

6. Creuzet $S$, Vincent $C$, Couly $G$. Neural crest derivatives in ocular and periocular structures. Int J Dev Biol $2005 ; 49$ : 161-71.

7. Lumsden AGS. Spatial organization of the epithelium and the role of neural crest cells in the initiation of the mammalian tooth germ. Development 1988 ; $103: 155-70$.

8. Le Douarin NM, Couly G, Creuzet SE. The neural crest is a powerful regulator of pre-otic brain development. Dev Biol $2012 ; 366: 74-82$

9. Etchevers HC, Dupin $\varepsilon$, Le Douarin NM. The diverse neural crest: from embryology to human pathology. Development 2019 ; 146. doi : 10.1242/dev.169821.

10. Jacob F. L'irrésistible ascension des gènes Hox. Med/ Sci (Paris) $1994 ; 10: 145-8$.

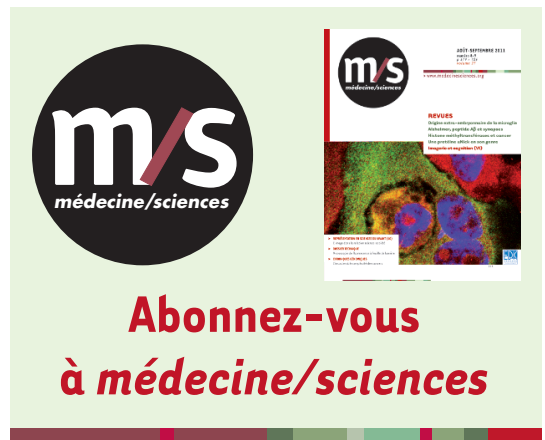

Bulletin d'abonnement page 594 dans ce numéro de $\mathrm{m} / \mathrm{s}$ 